\title{
NON-LINEAR EFFECTS IN LAMINAR FLOW \\ OVER WAVY SURFACES
}

\author{
PhiLIP JOHN KaChOYAN
}

We examine linear and non-linear effects in laminar flows over several types of wavy surfaces for various régimes of the wave amplitude $\varepsilon$ and the Reynolds number $R$. In particular, we are interested in the surface pressure and shear stress, which are important in considering wave growth.

In Section 1 we extend linear theory for parallel flows to include both third-order terms and finite Reynolds numbers. The Fourier-type expansion in $\varepsilon$ produces forced Orr-Sommerfeld problems which are solved numerically for various values of $\varepsilon$ and $R$. With increasing $\varepsilon$, the surface stress profiles become distorted from sinusoids and the location of the surface extrema migrate from their linear positions. Effects of 'fluid' boundary conditions, as opposed to 'solid' and 'flexible sheet' conditions are examined. The stress contours reveal elevated extrema out out of phase with the surface values. It is shown in two ways that an expansion of the above type requires $\varepsilon^{2} R^{4 / 3} \ll 1$, a much stricter condition than previously recognized.

In Section 2 we examine perturbations to the Blasius flow over a flat plate caused by two types of disturbances on the plate. Calculations are performed in parabolic co-ordinates $(\sigma, \tau)$ and expansions made in powers of $\varepsilon$ and then in powers of $R^{-\frac{1}{2}}$. The inner and outer solutions of the

Received 19 August 1983. Thesis submitted to University of New South Wales, April 1983. Degree approved August 1983. Supervisors: Dr N.G. Barton, Dr P.J. Blennerhassett.

Copyright Clearance Centre, Inc. Serial-fee code: 0004-9727/83 $\$ A 2.00+0.00$. 
resulting matched asymptotic expansion scheme are solved to $o\left(\varepsilon^{2}\right)$. The surface stresses exhibit different behaviour to those of section 1 and we obtain the restriction $\varepsilon \ll x^{\frac{1}{2}} \varepsilon<0.08$ for the expansion to be valid.

To try and overcome this severe restriction, triple-deck scalings for the wave amplitude and wavelength are assumed in section 3 , eventually reducing the problem to solving the lower deck equations. Analytical results are obtained for the linearized case, and then the full non-linear equations are solved numerically via a specially modified spectral method. Some of the linear results of Section 1 are derived as a limiting case of the linear theory. We study effects of increasing non-linearity and distance downstream and examine the resulting asymmetries, the behaviour near the leading edge of the disturbance and the onset of separation. Finally, it is shown that the first term in the lower deck expansion is enough to provide the first two terms in the main deck.

\author{
School of Mathematics, \\ University of New South Wales, \\ PO Box 1, \\ Kensington, \\ New South Wales 2033, \\ Australia.
}

